in IQ was found between patients with right-sided or left-sided hemiparesis whereas clinical seizures were more cammon in those with right-sided hemiparesis. Clinical epilepsy was related to impaired intellectual performance. Abnormal paroxysmal EEGs were more frequent with left-sided hemiparesis. Among those with clinical epilepsy a lower IQ was found in almost all with left-sided hemiparesis and almost half of those with right-sided hemiparesis. (Sussova $J$ et al. Hemiparetic forms of cerebral palsy in relation to epilepsy and mental retardation. Dev Med Child Neurol Sept 1990; $\underline{32}: 792-795)$.

CONMENT. A lower IQ may be expected in CP patients with clinical epilepsy, especially in those with left-sided hemiparesis. EEG abnormalities alone are not associated with a lower IQ.

\title{
BLOOD DISORDERS
}

IRON DEFICIENCY ANEMIA AND NEUROLOGIC DEFICITS

A 14 year old black female adolescent with focal neurological abnormalities complicating severe iron deficiency anemia is reported from Duke University Medical Center, Durham, North Carolina. The anemia was caused by bleeding from generalized intestinal polyposis and hereditary hemorrhagic telangiectasia complicated by nasal and gingival bleeding. Neurologic symptams and signs began with occipital headache and neck pain, intermittent diplopia, transient right-sided numbness and weakness, and a brief syncopal episode. On admission the patient was somnolent and the neurological examination revealed bilateral VI nerve palsies, facial palsies, papilledema, and generalized muscle weakness with normal reflexes. After transfusion with packed erythrocytes and treatment with ferrous sulfate orally the facial palsy resolved within 12 hours and the VI nerve palsy and somnolence resolved by the fifth day. A normal hemoglobin was maintained by iron supplementation and the neurologic exam remained normal despite continued gingival and nasal bleeding from telangiectases. (Bruggers CS et al. Reversible focal neurologic deficits in severe iron deficiency anemia. J Pediatr Sept 1990; $117: 430-432$ ).

COMMENT. An iron deficiency anemia is reported in $23 \%$ of cases of breath-holding spells in infants and young children. (Holowach J, Thurston DL. N Eng J Med 1963; 268:21). The neurologic abnormalities with ${ }^{-}$iron deficiency anemia may result from tissue hypoxia, increased capillary permeability, cerebral edema, and abnormal cytochrame enzyme function involved in oxygen metabolism.

VALPROATE-INDUCED CYTOPENIAS

A 16 year old white boy with trisomy 21 and valproic-acid induced erythrocyte aplasia is reported fram the Divisions of Hematology and Oncology, University of Alabama, Birmingham, AL. Suppression of hematopoiesis was demonstrated by in vitro studies of colony-forming- 
unit granulocyte/macrophage (CFU-GM) assays using bone marrow from healthy adult volunteers cultured in the presence of increasing doses of valproic acid $(60,120$, and $240 \mathrm{mcg} / \mathrm{ml})$. At a VPA concentration of $120 \mathrm{mcg} / \mathrm{ml}$, similar to that observed in the patient with erythrocyte aplasia, macrocytosis and neutropenia, there was a $67 \%$ CFU-GM growth inhibition, and at VPA levels of $240 \mathrm{mcg} / \mathrm{ml}, 84 \%$ of the colony growth was inhibited. The inhibition was specific to VPA and was not related to a change in $\mathrm{pH}$. The addition of the patient's serum to the assays had no significant effect. The results suggested a direct dose dependent suppression of bone marrow neutrophilic progenitors by valproic acid. (Watts RG et al. Valproic acid-induced cytopenias: Evidence for a dose-related suppression of hematopoiesis. J Pediatr Sept $1990 ; 117: 495-499)$.

COMMENT. This patient had no family history or medical history of anemia, congenital or acquired bone marrow failure, or malignancies. The only recent drug exposure was valproic acid. The authors recommend close hematologic monitoring of patients receiving valproic acid therapy and especially when larger doses are employed.

Of practical importance in the management of a severe overdose of valproic acid, studies of the elimination half life and clearance of valproic acid in a patient with dialysis-induced encephalopathy who was taking divalproic sodium for a seizure disorder showed that hemodialysis and hemoperfusion had little effect on the removal of valproic acid from the body. The equilibrium shifted so that valproic acid redistributed back into the blood from the tissues. (Kandrotas RJ et al. Neurology Sept $1990 ; 40: 1456-1458)$. Hemodialysis is unlikely to benefit patients with toxic overdose of valproic acid.

\section{FACTOR XI II DEFICIENCY AND INTRACRANIAL HEMORRHACE}

A 38 month old boy with excessive bleeding following circumcision as a newborn and two episodes of intracranial hemorrhage at four months and at $8 \frac{1}{2}$ months of age is reported from the Scott and White Clinic, Temple, TX. The diagnosis of Factor XIII deficiency was made in infancy and the bleeding was stopped with cryoprecipitate. Since 11 months of age he was treated with Fibrogammin at four week intervals and no further hemorrhages had occurred. MRI at three years demonstrated a left temporoparietal encephalomalacia and no vascular malformations. (Larsen PD et al. Factor XIII deficiency and intracranial hemorrhages in infancy. Pediatr Neurol July-August 1990; $\underline{6}: 277-278$ ).

OOMMENT. Repeated intracranial hemorrhage can occur spontaneously and without evidence of trauma in infants with Factor XIII deficiency. Routine coagulation studies do not detect this factor deficiency and examination for Factor XIII may avoid a misdiagnosis of child abuse. Replacement therapy should be maintained throughout life. 